\title{
Front Matter: Volume 8965
}

, "Front Matter: Volume 8965," Proc. SPIE 8965, High-Power Diode Laser Technology and Applications XII, 896501 (26 March 2014); doi: 10.1117/12.2062798

SPIE. Event: SPIE LASE, 2014, San Francisco, California, United States 


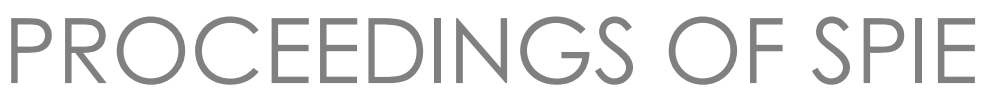

\section{High-Power Diode Laser Technology and Applications XII}

Mark S. Zediker

Editor

2-4 February 2014

San Francisco, California, United States

Sponsored and Published by

SPIE 
The papers included in this volume were part of the technical conference cited on the cover and title page. Papers were selected and subject to review by the editors and conference program committee. Some conference presentations may not be available for publication. The papers published in these proceedings reflect the work and thoughts of the authors and are published herein as submitted. The publisher is not responsible for the validity of the information or for any outcomes resulting from reliance thereon.

Please use the following format to cite material from this book:

Author(s), "Title of Paper," in High-Power Diode Laser Technology and Applications XII, edited by Mark S. Zediker, Proceedings of SPIE Vol. 8965 (SPIE, Bellingham, WA, 2014) Article CID Number.

ISSN: 0277-786X

ISBN: 9780819498786

Published by

SPIE

P.O. Box 10, Bellingham, Washington 98227-0010 USA

Telephone +1 3606763290 (Pacific Time) · Fax +1 3606471445

SPIE.org

Copyright $@ 2014$, Society of Photo-Optical Instrumentation Engineers.

Copying of material in this book for internal or personal use, or for the internal or personal use of specific clients, beyond the fair use provisions granted by the U.S. Copyright Law is authorized by SPIE subject to payment of copying fees. The Transactional Reporting Service base fee for this volume is $\$ 18.00$ per article (or portion thereof), which should be paid directly to the Copyright Clearance Center (CCC), 222 Rosewood Drive, Danvers, MA 01923. Payment may also be made electronically through $\mathrm{CCC}$ Online at copyright.com. Other copying for republication, resale, advertising or promotion, or any form of systematic or multiple reproduction of any material in this book is prohibited except with permission in writing from the publisher. The CCC fee code is 0277-786X/14/\$18.00.

Printed in the United States of America.

Publication of record for individual papers is online in the SPIE Digital Library.

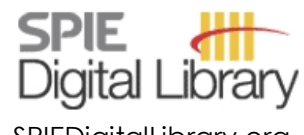

SPIEDigitalLibrary.org

Paper Numbering: Proceedings of SPIE follow an e-First publication model, with papers published first online and then in print and on CD-ROM. Papers are published as they are submitted and meet publication criteria. A unique, consistent, permanent citation identifier (CID) number is assigned to each article at the time of the first publication. Utilization of CIDs allows articles to be fully citable as soon as they are published online, and connects the same identifier to all online, print, and electronic versions of the publication. SPIE uses a six-digit CID article numbering system in which:

- The first four digits correspond to the SPIE volume number.

- The last two digits indicate publication order within the volume using a Base 36 numbering

system employing both numerals and letters. These two-number sets start with 00, 01, 02, 03, 04,

$05,06,07,08,09,0 A, 0 B \ldots$. OZ, followed by 10-1Z, 20-2Z, etc.

The CID Number appears on each page of the manuscript. The complete citation is used on the first page, and an abbreviated version on subsequent pages. Numbers in the index correspond to the last two digits of the six-digit CID Number. 


\section{Contents}

ix Conference Committee

\section{SESSION 1 HIGH POWER DIODE LASER RELIABILITY I}

896502 Reliability study on high-power $638 \mathrm{~nm}$ broad stripe LD with a window-mirror structure [8965-1]

T. Yagi, H. Mitsuyama, T. Nishida, K. Kadoiwa, K. Kuramoto, Mitsubishi Electric Corp. (Japan)

896503 Internal degradation of $980 \mathrm{~nm}$ emitting single-spatial-mode lasers during ultrahigh power operation [8965-2]

J. W. Tomm, M. Hempel, T. Elsaesser, Max-Born-Institut für Nichtlineare Optik und

Kurzzeitspektroskopie (Germany); J. Jimenez, V. Hortelano, Univ. de Valladolid (Spain); M. Bettiati, 3S PHOTONICS S.A.S. (France)

896504 Catastrophic degradation in high power multi-mode InGaAs-AIGaAs strained quantum well lasers with intrinsic and irradiation-induced defects [8965-3]

Y. Sin, S. LaLumondiere, E. Delonno, B. Foran, N. Presser, W. Lotshaw, S. C. Moss, The Aerospace Corp. (United States)

896505 Nanoscale coatings for erosion and corrosion protection of copper microchannel coolers for high powered laser diodes [8965-4] M. Flannery, A. Fan, T. G. Desai, Advanced Cooling Technologies, Inc. (United States)

896506 Feedback-induced catastrophic optical mirror damage (COMD) on 976nm broad area single emitters with different AR reflectivity [8965-5]

B. Leonhäuser, H. Kissel, A. Unger, B. Köhler, J. Biesenbach, DILAS Diodenlaser GmbH (Germany)

\section{SESSION 2 HIGH POWER DIODE LASER RELIABILITY II}

896507 Advancements in laser diode chip and packaging technologies for application in kWclass fiber laser pumping [8965-6]

E. Zucker, D. Zou, L. Zavala, H. Yu, P. Yalamanchili, L. Xu, H. Xu, D. Venables, J. Skidmore, V. Rossin, R. Raju, M. Peters, K.-H. Liao, K.-W. Lee, B. Kharlamov, A. Hsieh, R. Gurram, J. Guo, N. Guerin, J. Gregg, R. Duesterberg, J. Du, A. Demir, P. Cheng, J. Cheng, JDSU (United States); H. Ishiguro, R. Li, Y. Mizoguchi, H. Sako, Amada Co., Ltd. (Japan)

896508 High reliability of high power and high brightness diode lasers [8965-46] M. Kanskar, L. Bao, J. Bai, Z. Chen, D. Dahlen, M. DeVito, W. Dong, M. Grimshaw, J. Haden, X. Guan, M. Hemenway, K. Kennedy, R. Martinsen, J. Tibbals, W. Urbanek, S. Zhang, nLIGHT Corp. (United States) 
896509 Watt-level continuous-wave diode lasers at $1180 \mathrm{~nm}$ with InGaAs quantum wells [8965-8]

K. Paschke, F. Bugge, G. Blume, D. Feise, W. John, S. Knigge, M. Matalla, H. Wenzel,

G. Erbert, Ferdinand-Braun-Institut (Germany)

\section{SESSION 3 HIGH POWER BEAM COMBINING I}

8965 OB A 25kW fiber-coupled diode laser for pumping applications [8965-10]

J. Malchus, V. Krause, A. Koesters, Laserline GmbH (Germany); D. G. Matthews, Laserline Inc. (United States)

8965 OC High-power dense wavelength division multiplexing (HP-DWDM) of frequency stabilized 9xx diode laser bars with a channel spacing of $1.5 \mathrm{~nm}$ [8965-11]

S. Hengesbach, C. Holly, RWTH Aachen Univ. (Germany); N. Krauch, U. Witte,

T. Westphalen, M. Traub, D. Hoffmann, Fraunhofer-Institut für Lasertechnik (Germany)

8965 OD High-power fiber-coupled diode lasers with superior brightness, efficiency, and reliability [8965-12]

K. Kennedy, M. Hemenway, W. Urbanek, K. Hoener, K. Price, L. Bao, D. Dawson, M. Kanskar, J. Haden, nLIGHT Corp. (United States)

8965 OF Integrated coherent beam combining of a laser diode mini-bar [8965-14]

Y. Zhao, L. Zhu, Clemson Univ. (United States)

SESSION 4 HIGH POWER BEAM COMBINING II

8965 0G High brightness direct diode laser with kW output power [8965-15]

H. Fritsche, B. Kruschke, R. Koch, F. Ferrario, H. Kern, U. Pahl, DirectPhotonics Industries

GmbH (Germany); S. Pflueger, DirectPhotonics Inc. (United States); W. Gries,

DirectPhotonics Industries GmbH (Germany)

8965 Ol Narrow-stripe broad-area lasers with distributed-feedback surface gratings as brilliant sources for high power spectral beam combining systems [8965-17]

J. Decker, P. Crump, J. Fricke, H. Wenzel, A. Maaßdorf, G. Erbert, G. Tränkle, Ferdinand-

Braun-Institut (Germany)

8965 0J Scalable and modular diode laser architecture for fiber coupling that combines highpower, high-brightness, and low weight [8965-18]

A. Bayer, B. Köhler, A. Noeke, M. Küster, D. Irwin, S. Patterson, J. Biesenbach, DILAS

Diodenlaser $\mathrm{GmbH}$ (Germany)

8965 OK Numerical analysis of external feedback concepts for spectral stabilization of high-power broad-area semiconductor lasers [8965-19]

C. Holly, S. Hengesbach, RWTH Aachen (Germany); M. Traub, D. Hoffmann, FraunhoferInstitut für Lasertechnik (Germany) 
8965 OM High-brightness 800nm fiber-coupled laser diodes [8965-21]

Y. Berk, M. Levy, N. Rappaport, R. Tessler, O. Peleg, M. Shamay, D. Yanson, G. Klumel, N. Dahan, I. Baskin, L. Shkedi, SCD SemiConductor Devices (Israel)

8965 ON High-volume manufacturing of $8 X X \mathrm{Xnm}-10 \mathrm{XXnm}$ single emitter pumps by MBE growth technique [8965-22]

V. Gapontsev, N. Moshegov, I. Berezin, P. Trubenko, A. Komissarov, D. Miftakhutdinov, I. Berishev, N. Strougov, V. Chuyanov, O. Raisky, A. Ovtchinnikov, IPG Photonics Corp. (United States)

896500 Study of waveguide designs for high-power 9xx-nm diode lasers operating at $200 \mathrm{~K}$ [8965-23]

C. Frevert, P. Crump, F. Bugge, S. Knigge, G. Erbert, Ferdinand-Braun-Institut (Germany)

8965 OP Two photon absorption in high power broad area laser diodes [8965-24]

M. Dogan, Science Research Lab., Inc. (United States); C. P. Michael, Y. Zheng, Booz Allen Hamilton Inc. (United States); L. Zhu, Clemson Univ. (United States); J. H. Jacob, Science Research Lab., Inc. (United States)

$89650 Q \quad$ High differential efficiency tilted wave laser [8965-25]

N. N. Ledentsov, V. A. Shchukin, VI Systems GmbH (Germany) and Ioffe Physico-Technical Institute (Russian Federation); N. Yu. Gordeev, Yu. M. Shernyakov, A. S. Payusov, M. V. Maximov, Ioffe Physico-Technical Institute (Russian Federation) and St. Petersburg Academic Univ. (Russian Federation); N. A. Kaluzhniy, S. A. Mintairov, V. M. Lantratov, K. A. Vashanova, M. M. Kulagina, Ioffe Physico-Technical Institute (Russian Federation); S. Rouvimov, Univ. of Notre Dame (United States)

\section{SESSION 6 HIGH POWER DEVICES II}

8965 OR High power pump laser diodes for $2 \mu \mathrm{m}$ fiber laser [8965-26]

S. Pawlik, R. Todt, M. Moser, O. Romero, N. Lichtenstein, II-VI Laser Enterprise GmbH (Switzerland)

8965 OS Wavelength stabilization of high power laser systems using volume holographic gratings [8965-27]

V. C. Negoita, Y. Li, T. Barnowski, J. Jiang, H. An, R. Roff, M. Shih, T. Vethake, TRUMPF Photonics (United States); T. Gottwald, S. Schad, TRUMPF Laser GmbH \& Co. KG (Germany); G. Treusch, TRUMPF Photonics (United States)

8965 OT New highly efficient laser bars and laser arrays for $\mathbf{8 x x}-10 x \mathbf{n m}$ pumping applications [8965-28]

A. Pietrzak, R. Huelsewede, M. Zorn, O. Hirsekorn, J. Sebastian, JENOPTIK Diode Lab. GmbH (Germany); J. Meusel, V. Bluemel, P. Hennig, JENOPTIK Laser GmbH (Germany)

8965 OU Methods for slow axis beam quality improvement of high power broad area diode lasers [8965-29]

H. An, Y. Xiong, C.-L. Jiang, TRUMPF Photonics (United States); B. Schmidt, TRUMPF Laser Marking Systems AG (Switzerland); G. Treusch, TRUMPF Photonics (United States) 
8965 OV Automated alignment of fast-axis collimator lenses for high-power diode laser bars [8965-30]

T. Westphalen, Fraunhofer-Institut für Lasertechnik (Germany); S. Hengesbach, C. Holly, RWTH Aachen Univ. (Germany); M. Traub, D. Hoffmann, Fraunhofer-Institut für Lasertechnik (Germany)

8965 0W Aplanatic beam shaping for diffraction limited beam circularization of tapered laser diodes [8965-31]

A. Heinrich, C. Hagen, M. Harlander, B. Nussbaumer, Pantec Engineering AG (Liechtenstein)

8965 OY Robust adhesive precision bonding in automated assembly cells [8965-33]

T. Müller, S. Haag, T. Bastuck, Fraunhofer-Institut für Produktionstechnologie (Germany); T. Gisler, H. Moser, FISBA OPTIK AG (Switzerland); P. Uusimaa, Modulight, Inc. (Finland); C. Axt, Rohwedder Micro Assembly GmbH (Germany); C. Brecher, Fraunhofer-Institut für Produktionstechnologie (Germany)

\section{SESSION $8 \quad$ HIGH POWER DEVICES III}

896510 High power laser diodes at 14xx-nm wavelength range for industrial and medical applications [8965-35]

J. Telkkälä, J. Boucart, M. Krejci, T. Crum, N. Lichtenstein, II-VI Laser Enterprise GmbH (Switzerland)

896511 High power and high efficiency 14xx-nm wavelength Fabry-Perot lasers [8965-36] T. Tanbun-Ek, R. Pathak, Z. Wang, H. Winhold, S. Kim, Coherent Inc. (United States)

896512 High power diode lasers emitting from $639 \mathrm{~nm}$ to $690 \mathrm{~nm}$ [8965-37]

L. Bao, M. Grimshaw, M. DeVito, M. Kanskar, W. Dong, X. Guan, S. Zhang, J. Patterson, P. Dickerson, K. Kennedy, S. Li, J. Haden, R. Martinsen, nLIGHT Corp. (United States)

896513 High-power visible spectrum diode lasers for display and medical applications: beam sources with tailored beam quality and spectral characteristics [8965-38]

A. Unger, B. Köhler, J. Biesenbach, DILAS Diodenlaser GmbH (Germany)

896514 High power and high efficiency kW 88x-nm multi-junction pulsed diode laser bars and arrays [8965-39]

Z. Chen, J. Bai, W. Dong, X. Guan, S. Zhang, S. Elim, L. Bao, M. Grimshaw, M. Devito, M. Kanskar, nLIGHT Corp. (United States)

896515 Simple design for fiber coupled 9xx-nm kW-QCW pump module with high duty cycle based on customized chips and lateral heat removal [8965-40]

W. Pittroff, B. Eppich, G. Erbert, R. Platz, Ferdinand-Braun-Institut (Germany); D. Tyralla, Technische Hochschule Wildau (Germany); G. Tränkle, Ferdinand-Braun-Institut (Germany) 
896516 Characterization of diode-laser stacks for high-energy-class solid state lasers [8965-7] J. Pilar, P. Sikocinski, A. Pranowicz, M. Divoky, Institute of Physics of the ASCR, v.v.i. (Czech Republic); P. Crump, R. Staske, Ferdinand-Braun-Institut (Germany); A. Lucianetti,

T. Mocek, Institute of Physics of the ASCR, v.v.i. (Czech Republic)

896518 Laser technology in automotive lighting [8965-43]

C. Altingöz, Istanbul Technical Univ. (Turkey) and Automotive Lighting TR-Mako (Turkey)

896519 High-resolution spectral mapping of a lensed high power laser bar [8965-45]

C. D. Gannon, Rose-Hulman Institute of Technology (United States); T. Koenning,

S. G. Patterson, DILAS Diode Laser, Inc. (United States); P. O. Leisher, Rose-Hulman Institute of Technology (United States)

89651 A Coupling of DBR tapered diode laser radiation into a single-mode-fiber at high powers [8965-47]

D. Jedrzejczyk, Ferdinand-Braun-Institut (Germany); P. Asbahr, Ferdinand-Braun-Institut (Germany) and FCC FiberCableConnect GmbH (Germany); M. Pulka, FCC

FibreCableConnect GmbH (Germany); B. Eppich, K. Paschke, Ferdinand-Braun-Institut (Germany)

8965 IB Design and fabrication of high power single mode double-trench ridge waveguide laser [8965-48]

S. Tan, T. Zhai, Institute of Semiconductors (China); W. Wang, R. Zhang, D. Lu, C. Ji, Institute of Semiconductors (China)

Author Index 
Proc. of SPIE Vol. $8965896501-8$

Downloaded From: https://www.spiedigitallibrary.org/conference-proceedings-of-spie on 25 Apr 2023 Terms of Use: https://www.spiedigitallibrary.org/terms-of-use 


\title{
Conference Committee
}

\author{
Symposium Chairs
}

Bo Gu, Bos Photonics (United States)

Andreas Tünnermann, Fraunhofer-Institut für Angewandte Optik und Feinmechanik (Germany) and Friedrich-Schiller-Universität Jena (Germany)

Symposium Co-chairs

Guido Hennig, Daetwyler Graphics AG (Swtizerland)

Yongfeng Lu, University of Nebraska-Lincoln (United States)

Program Track Chair

Klaus P. Streubel, OSRAM AG (Germany)

Conference Chair

Mark S. Zediker, Foro Energy, Inc. (United States)

Conference Program Committee

Friedrich G. Bachmann, FriBa LaserNet (Germany)

Stefan W. Heinemann, TRUMPF Photonics (United States)

Volker Krause, Laserline GmbH (Germany)

Robert J. Martinsen, nLIGHT Corporation (United States)

Kurt J. Linden, Spire Corporation (United States)

Erik P. Zucker, JDSU (United States)

\section{Session Chairs}

1 High Power Diode Laser Reliability I

Erik P. Zucker, JDSU (United States)

2 High Power Diode Laser Reliability II

Erik P. Zucker, JDSU (United States)

3 High Power Beam Combining I

Stefan W. Heinemann, TRUMPF Photonics (United States)

$4 \quad$ High Power Beam Combining II

Volker Krause, Laserline GmbH (Germany) 
5 High Power Devices I

Rob Martinsen, nLIGHT Corporation (United States)

6 High Power Devices II

Rob Martinsen, nLIGHT Corporation (United States)

7 High Power Diode Laser Optics

Stefan W. Heinemann, TRUMPF Photonics (United States)

8 High Power Devices III

Kurt J. Linden, Spire Corporation (United States) 